\title{
Lung ultrasonography: A new imaging approach in diagnosis of pneumonia in children
}

\author{
Damjana Ključevšek ${ }^{*}$ \\ University Medical Center, Children's Hospital \\ Radiology Unit, Ljubljana, Slovenia \\ ${ }^{\star}$ Corresponding author: \\ damjana.kljucevsek@gmail.com \\ Tel.: + 38615229264 \\ Fax.: + 38615222993
}

Received/Accepted: 2 May 2016

Key words: Lung ultrasonography - Pneumonia Children.

Respiratory tract infections are a significant cause of pediatric morbidity and mortality. Chest radiography is the most common diagnostic imaging. Current guidelines suggest that a diagnosis of pneumonia can only be made considering clinical history, respiratory rate, fever, respiratory signs and symptoms limited the use of radiography to severe or complicated cases only (1). Up till now lung ultrasound (LUS) has not been mentioned as a diagnostic tool in current guidelines.

Although LUS is not in the guidelines, it has gained a value in the clinical practice in the last 15 years. It moved from the traditional assessment of pleural effusions and thoracic masses towards the imaging of the pulmonary parenchyma, mainly as a pointof-care technique (2). As a technique LUS has many advantages. It is quick to preform, repeatable, and radiation-free compared to chest radiography or computer tomography. It has been proved that the learning curve of LUS is relatively short ("easy-to-learn technique"), less technically demanding than other sonographic examinations. In addition, the main advantage of LUS in children is related to the small size of the chest, which allows quite optimal (still indirect) visualization of the lungs by the point-ofcase technique. However, ultrasonographers should be aware of its limitations. US waves are reflected by alveolar air and bones of the thoracic cage. Pulmonary changes are detected only when the lesion reaches the lung surface. Pathological findings within hilar regions are missed, and there is a problem in detection of air-filled lung abscesses. Despite these limitations LUS has proved to be useful in the evaluation of many clinical pulmonary conditions, acute or chronic, in children and adults.

Most of the published studies compared chest radiography with LUS in pediatric population. It was shown that LUS is especially valuable in detecting pneumonia in children, with sensitivity even higher than that of chest radiography. A meta-analysis including 8 pediatric studies comparing LUS with X-ray was performed by Pereda et al. (3); LUS had a sensitivity of $96 \%$ and specificity of $93 \%$, and positive and negative likelihood ratios were 15.3 and 0.06 , respectively. 
Lovrenski et al. (4) presented a unique prospective study comparing LUS and lung auscultation using stetoscope, a widely accepted diagnostic tool. The technique of LUS is a well described, standardized using trans-thoracic and transabdominal approach. In experienced hands the examination is relatively short (average time 5.7 minutes). Of course, the examination time depends on the size of the thorax, a child's cooperation and the complexity of the findings. The wide range of positive findings depending on the stage of pneumonia at the time of examination is also well described and explained. LUS has proved to show more positive findings compared to auscultation in children with suspected pneumonia. A very important scientific contribution is the determination of the threshold size of subpleural consolidations below which it is highly unlikely that pneumonia will be detected by auscultation: a cranio-caudal size of subpleural consolidation of less than 30 $\mathrm{mm}$ significantly reduced the possibility of an auscultatory detection. The limitations of LUS and study were discussed.

How can LUS be introduced in diagnostic algorithm in children with suspected pneumonia? Is its place in diagnostical algorithms? Iorio et al. proposed LUS as initial imaging in children with suspected pneumonia (5). According to the published data by Lovrenski et al. (4) LUS should be used whenever physical findings are unclear and in cases with a negative auscultation and strong clinical suspicion of pneumonia. Consequently, children would not be exposed to unnecessary chest radiography.

In conclusion, LUS is a promising method in the evaluation of children with suspected pneumonia, with many advantages and some limitations, which should be respected. LUS has a great potential to be implemented in everyday clinical practice in detecting and following pneumonia and some of its complications. Its role should be further evaluated. Every study from that field contributes a piece in the mosaic. In the future, there is hope that the introduction of LUS in diagnostic algorithm will diminish the use of radiation to a certain degree, which is in concordance with the nowadays strategy regarding radiation in children.

Conflict of interest: The author declares that she has no conflict of interest.

\section{References}

1. Bradley JS, Byington CL, Shah SS, Alverson B, Carter ER, Harrison C et al. Executive summary: the management of community-acquired pneumonia in infants and children older than 3 months of age: clinical practice guidelines by the Pediatric Infectious Diseases Society and the Infectious Diseases Society of America. Clin Infect Dis. 2011; 53(7):617-30.

2. Volpicceli G. Lung Sonography. J Ultrasound Med. 2013;32:165-71.

3. Pereda MA, Chavez MA, Hooper-Miele CC, Gilman RH, Steinhoff MC, Ellington LE et al. Lung ultrasound for the diagnosis of pneumonia in children: a meta-analysis. Pediatrics. 2015;135(4):71422.

4. Lovrenski J, Petrović S, Balj-Barbir S, Jokić R, Vilotijević-Dautović G. Stetoscope vs. ultrasound probe - which is more reliable in children with suspected pneumonia? Acta Med Acad. 2016;45(1):39-50.

5. Iorio G, Capasso M, De Luca G, Prisco S, Mancusi $\mathrm{C}$, Laganà $\mathrm{B}$ et al. Lung ultrasound in the diagnosis of pneumonia in children: proposal for a new diagnostic algorithm. PeerJ. 2015;3:e1374. 